\title{
Empirical Study of English Teaching Model Based on Flipped Teaching Yuting Fan ${ }^{1, a}$ and Yang Luo ${ }^{2, b}$
}

${ }^{1}$ Foreign language school affiliated to Sichuan Foreign Language University, Chongqing, 400039, China

${ }^{2}$ Medical Research Center, Southwest Hospital, Third Military Medical University, Chongqing, 400038, China

a290021405@qq.com, bluoyang@tmmu.edu.cn

Keywords: English learning; Flipped teaching; Empirical study; Educational reform

Abstract. English teaching in China has come a long way since pedagogical reform. Meanwhile, there are still some existing problems in the idea of teaching reform and teaching conception, due to which flipped teaching are adopted and gradually popularized. In practice, we found both flipped teaching and traditional classroom have their virtues and faults by comparing them in teaching construction, teaching process and teaching efficiency. As a result, not only can we improve the ability of study independently, innovative thinking and cooperation of students, but also we can improve teaching effect and quality.

\section{基于翻转课堂的英语教学模式之实证研究}

\author{
樊玉婷 ${ }^{1, a}$, 罗阳 ${ }^{2, b}$ \\ 1. 四川外国语大学附属外国语学校, 中国重庆 400039 \\ 2. 第三军医大学第一附属医院综合实验研究中心, 中国重庆 400038

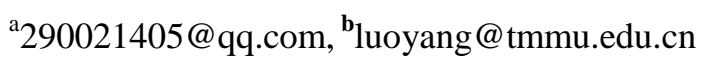

摘要: 自推行教学改革以来, 我国英语教学取得了长足、稳定的发展。但目前的英语教学依 然存在教师 “一言堂”、学生缺乏学习热情、教学效率低下的问题。鉴于此, 翻转课堂在我国 教学中得以采用并逐渐推广。在实践中, 笔者通过教学实例拟将翻转课堂与传统课堂分别从 教学结构、教学流程和教学效果方面加以对比分析, 发现二者各有利弊。因此, 如果将两种 模式有机结合, 相互渗透, 取长补短, 不仅可以培养学生课后独立学习和创新思维、实施小 组合作探究的能力, 而且可以提高教学质量, 优化教学效果, 加速推进我国的教学改革。

\section{关键词: 英语教学; 转课堂; 实证研究}

\section{1. 引言}

近年来, 由于翻转课堂具有新型的教育理念和教学模式, 并取得了良好的教学效果, 因此率 先在美国, 并迅速在世界多国都得以采用和推广。我国正处于教学改革的关键时期, 为了提 高教学效率, 不少学校开始尝试采用翻转课堂教学模式 [1]。然而, 翻转课堂在我国究竟会洋 为中用, 还是会水土不服呢? 在本文中, 笔者会就此进行深入探讨。

\section{2. 翻转课堂的内涵}

翻转课堂译自“Flipped Classroom” 或 “Inverted Classroom”, 一般被称为 “反转课堂式教学模 式”, 是指重新调整课内外的时间, 将学习的决定权从教师转移给学生。传统的教学模式是 老师在课堂上讲课, 布置家庭作业, 让学生回家练习 [2]。与传统的课堂教学模式不同, 在 “翻 转课堂式教学模式” 下, 学生在家完成知识的学习, 而课堂变成了老师学生之间和学生与学 
生之间互动的场所, 包括答疑解惑、知识的运用等, 从而达到更好的教育效果 [3]。互联网的 普及和计算机技术在教育领域的应用, 使 “翻转课堂式” 教学模式变得可行和现实。学生可 以通过互联网去使用优质的教育资源, 不再单纯地依赖授课老师去教授知识。而课堂和老师 的角色则发生了变化。老师更多的责任是去针对性地解决学生的问题和引导学生去运用知识。 在课后, 学生自主规划学习内容和学习进度, 集自主性学习、探究式学习为一体, 让学生成 为教学的主体, 变被动学习为主动学习 $[4]$ 。

\section{3. 在英语课堂中实施翻转课堂与传统课堂的教学对比}

为检验翻转课堂的科学性和有效性, 笔者大胆实践, 从所在单位选取两个教学班（这两个班 英语期末均分差距仅为 0.5 分) 分别实施 “翻转课堂教学模式” (简称实验班) 和 “传统课堂 教学模式”(简称对照班)。

3. 1. 教学结构对比 [2]

表 1 教学结构对比

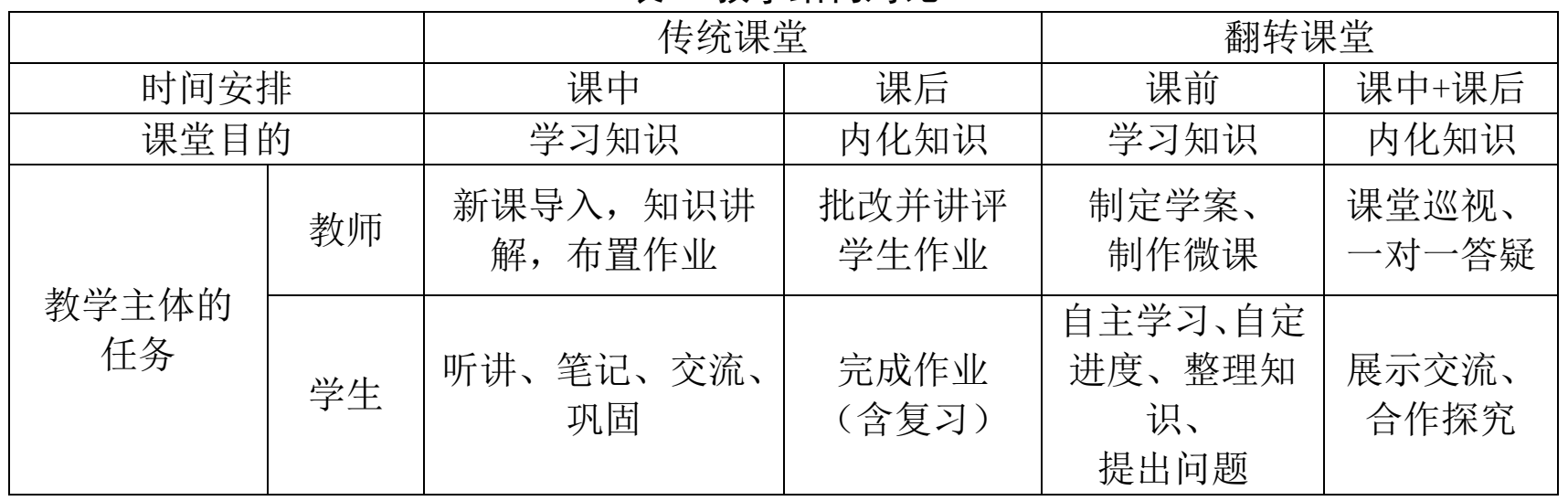

\section{2. 教学流程对比 [3]}

在 “虚拟语气” 的教学过程中, 笔者在对照班采用传统课堂教学模式, 如下图 (图 1) 所示。 在传统教学模式中, 笔者认真备课后, 首先在课堂上呈现出虚拟语气的若干实例, 然后让学 生分组讨论, 并归纳总结虚拟语气的用法规则, 然后在系统全面地介绍虚拟语气的定义、用 法、特殊句式等, 通过循循善诱让学生逐渐掌握虚拟语气的用法。笔者发现, 在教学过程中, 一定要给学生充足的时间来分组讨论并归纳总结虚拟语气的用法, 并且通过随堂练习及时为 学生查漏补缺。但难点在于, 由于课堂时间有限, 而课堂容量大, 相当一部分学生, 尤其是 基础较为薄弱的学生会感到 “消化” 困难, 加上特殊句式的出现, 学习难度倍增, 让学生的 学习积极性和自信心大打折扣, 直接影响了本课的教学效果。 


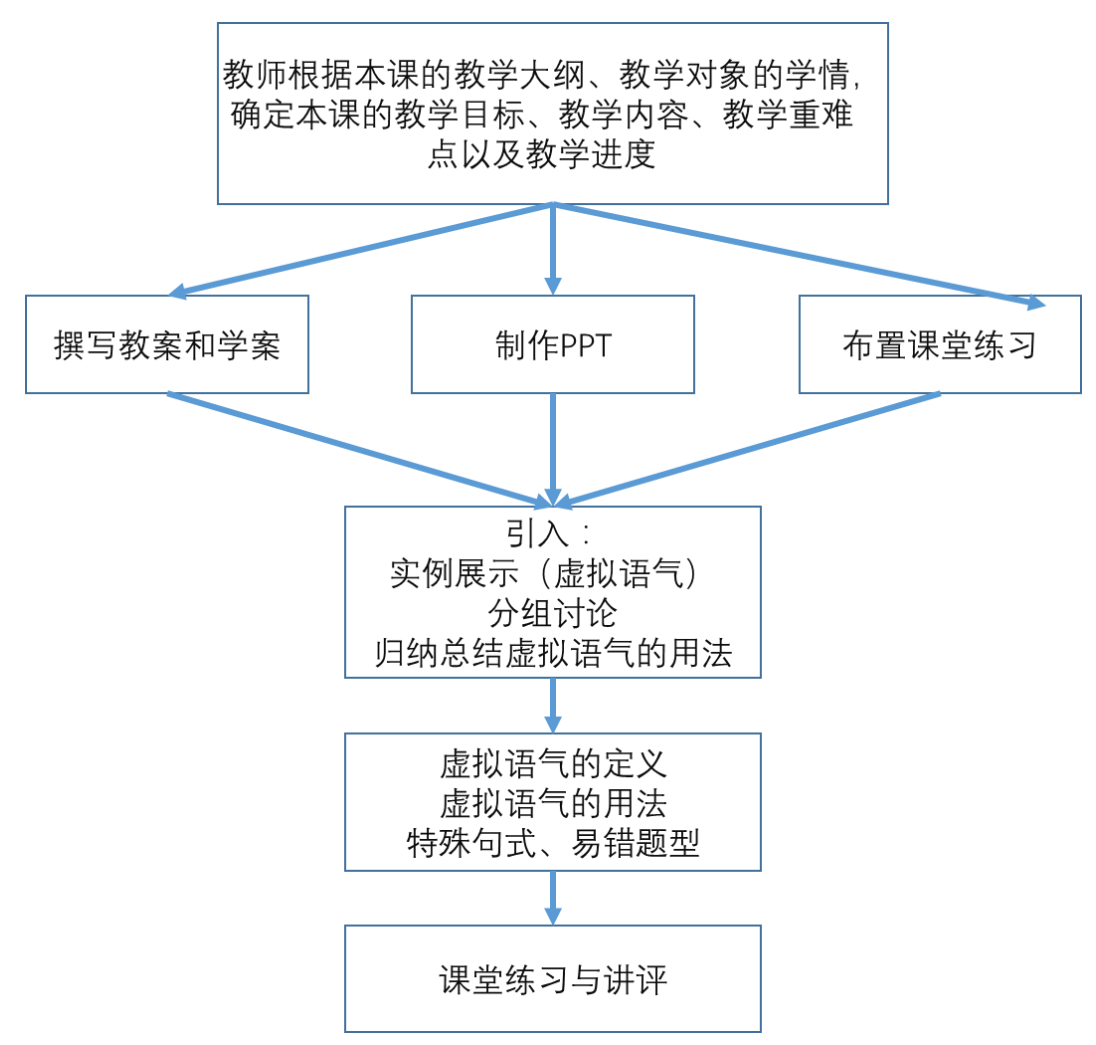

图 1 对照班采取传统教学模式



图 2 实验班班采用 “翻转课堂” 教学模式

笔者在实验班班采用翻转课堂教学模式 (图 2), 主要包括课前学习和课堂知识内化两个部分。 在备课时, 笔者设计了一套详尽的学案, 让学生明确本课的学习内容、目标, 并为其提供相 应的学习资源。学生可以根据自己的实际情况调整学习进度, 充分咀嚼并消化每个知识点, 最大程度地汲取微课视频的营养。对于自己无法解决的问题学生可以及时标记, 在课堂环节, 通过小组合作, 师生合作的形式进行解决。这样, 每一个学生都能摘到树上的苹果, 并且享 
受到苹果的甜美的滋味。

笔者发现, 通过翻转课堂的教学模式, 将课前的微课学习作为铺垫, 在有限的课堂时间内, 最大程度地解决难点和易错点, 使得学生的学习效率大大提高。

3.3. 教学效果利弊的对比

在讲授虚拟语气时, 采用翻转课堂和传统课堂教学模式, 各有利弊。

3. 3.1 翻转课堂教学的优点

第一, 翻转课堂能够满足不同水平学生的学习需求。

在虚拟语气的教学中, 时态的正确使用一直是其瓶颈。学习基础差, 领悟能力弱的学生更难 在短短四十分钟的课堂时间内完全掌握该语法点。然而, 在翻转课堂中, 这部分学生就可以 根据自己的学习情况, 实时暂停或回放视频, 反复学习知识点、听取老师的讲解, 通过重复 多次的学习逐渐掌握其重难点。同时, 老师也可以根据学生的自学情况, 有针对性地对学生 进行个别辅导答疑, 做到个性化教学。

第二, 翻转课堂解决了传统课堂无法解决的另一个问题, 即 “学生自主选择所学内容” [5]。 面对不同层次的学生, 教师的微课通常包含必学内容和选学内容。因此, 学生可以根据自己 的实际情况自行选择学习内容。虚拟语气的内容繁杂, 基础薄弱的学生可以选择容易的板块 学习; 而学有余力的学生可以尝试继续学习混合时态板块的内容, 以此培养学生良好的自学 习惯和能力。

第三, 翻转课堂使得 “一对一的个性化教学成为可能” [6]。

经过课前微课学习环节, 学生对于所学内容已经明确了重难点; 在课堂巡视过程中, 教师就 可以根据学生的具体学习情况, 实施 “一对一的个性化教学”，做到有的放矢。

3. 3.2 传统课堂教学的优点

第一, 传统课堂在探究课型上较翻转课堂有优势, 能够让学生亲身体会真实的探究过程, 有 助于培养学生的自学能力、创新开拓和团结协作的能力 [7]。例如, 在英语写作课 “在学校是 否应该禁用手机” 的传统教学中, 通常让学生事先就题目完成作文初稿, 然后在课堂上采用 小组互评机制, 分组讨论作文的优点和不足, 并经过集体修改后, 再到班级讲评。在课程中, 学生通过自我创作、小组互评、集体修改、归纳总结的步骤, 体会到每一个环节的有效性、

创造性和成就感。同时, 更能培养学生发现问题、提出问题、解决问题的能力, 提升学生的 思维层次, 同时让体验到学习的乐趣, 激发起高昂的学习热情和动力。

然而, 在该写作课的翻转课堂中, 尽管在学案中已提供了与话题相关的词汇、搭配和句型, 让学生能够在课前学习。但是在课堂中, 老师主要进行个别辅导与答疑, 课堂上没有充足的 时间让学生协作探究, 循序渐进, 从而不能达到探究型教学的效果。鉴于此, 笔者认为, 将 写作课的传统教学模式和翻转课堂教学模式有机融合, 相互取长补短, 教学效果会得到明显 改善。

第二，传统课堂在隐性知识的传授上比翻转课堂更有优势 $[8]$ 。

在翻转课堂中, 教师的微课视频是提前学习的, 尽管在授课过程中有时间停顿, 但是学生与 老师缺乏面对面的交流, 而且在课堂答疑的过程中, 老师往往把更多的精力花在 “一对一” 辅导上，从而在课堂节奏的把握上往往缺乏宏观性和全局性。

再者, 在听说课型中, 教师传授的不仅仅是显性知识, 还有教师个人的隐形知识蕴含其中, 这种隐性知识既包含教师的思维方式, 对知识的态度, 对学生的情感, 而且还包括教师的一 个动作, 一个微笑, 甚至是教师的人格鬼力 $[9,10]$ 。在翻转课堂中, 这些隐性知识的传播渠 道被截断, 学生在学习中的情感参与缺失了载体, 课堂教学在这方面的功能被削弱了。

\section{4. 教学反思与建议}

笔者在教学实践中发现, “翻转课堂教学模式” 和 “传统课堂教学模式” 各有优势, 也存在自 身的不足。倘若在教学中, 合理有效地将二者融合起来, 势必可以取得更好的教学效果。 例如在语法课中, 由于传授的知识容量大, 且抽象不易理解。对此, 教师可以利用微课视频 
让学生事先熟悉要学习的新知识, 比如定义、用法、实例等等; 另一方面, 学生也可以根据 自己的实际情况, 自主选择学习的次数; 在课堂环节, 有了课前的铺垫, 教师可以针对学生 的难点部分重点讲解, 并通过随堂练习让学生不断巩固强化。在 “活动与探究” 与” 观察与 思考” 的过程中，将 “翻转课堂” 和 “传统课堂” 巧妙结合起来，有的放矢，事半功倍。

\section{5. 结论}

其实, 在教学实践中, 往往教无定法。作为一线的教育者, 我们应当根据实际的教情与学 情, 将 “传统课堂教学模式” 和 “翻转课堂教学模式” 有机结合, 取长补短, 以期形成更高 效的教学模式, 让学生成为教学的主体, 尽情体验学习的乐趣, 实现 “要我学” 到 “我要 学” 以至 “我爱学” 的转变。从而进一步提高教学效率, 推动英语教学改革。

\section{6. 致谢}

本文感谢以下基金的资助: 中国学位与研究生教育学会研究课题（B2-2015Y0501-022); 重庆 市研究生教育教学改革研究项目 (yjg143006); 重庆市高等教育学会高等教育科学研究课题 (CQGJ15161C); 全军学位与研究生教育研讨会研究课题 (YJZX14D23)

\section{参考文献}

[1] Gregory Mason. Inverting (Flipping) Classrooms - Advantages and Challenges [Z]. 2013.

[2] Gilboy M B, Heinerichs S, Pazzaglia G. Enhancing student engagement using the flipped classroom[J]. Journal of nutrition education and behavior, 2015, 47(1): 109-114.

[3] Abeysekera L, Dawson P. Motivation and cognitive load in the flipped classroom: definition, rationale and a call for research [J]. Higher Education Research \& Development, 2015, 34(1): $1-14$.

[4] 吴华, 孙丽梅. 翻转课堂教学模式在数学教学中的应用[J]. 中小学信息技术教育, 2014(1): 21-25

[5] 汪晓东, 张晨婧. 翻转课堂在大学中的应用研究, 2013(8): 46

[6] Hurtubise L, Hall E, Sheridan L, et al. The flipped classroom in medical education: engaging students to build competency[J]. Journal of Medical Education and Curricular Development, 2015(2): 35-43.

[7] 金陵. 建构中国特色的微课程教学法[J]. 中国信息技术教育，2013（3）：20

[8] 张跃国, 张渝江. 透视 “翻转课堂”[J]. 中小学信息技术教育, 2012（3）：9-10

[9] 钟晓流. 信息化环境中基于翻转课堂理念的教育设计研究 [J]. 开放教育研究, 2013,19(2).

[10]梁乐明,曹俏俏,张宝辉. 微课程设计模式研究——基于国内外微课程的对比分析 [J]. 开放 教育研究, 2013,19(1).

\section{Acknowledgement}

This research was financially supported by the fund of the Chinese Society of Academic Degrees and Graduate Education (B2-2015Y0501-022); Graduate Education Reform fund of Chongqing (yjg143006); Educational Scientific fund of Chongqing Association of Higher Education (CQGJ15161C); and the project of Academic Degrees and Graduate Education Seminar (YJZX14D23). 


\section{References}

[1] Gregory Mason. Inverting (Flipping) Classrooms - Advantages and Challenges [Z]. 2013.

[2] Gilboy M B, Heinerichs S, Pazzaglia G. Enhancing student engagement using the flipped classroom[J]. Journal of nutrition education and behavior, Vol. 47 (2015), No.1, pp. 109-114.

[3] Abeysekera L, Dawson P. Motivation and cognitive load in the flipped classroom: definition, rationale and a call for research [J]. Higher Education Research \& Development, Vol. 34 (2015), No.1, pp. 1-14.

[4] Wu Hua, Sun Limei: "Application of Inverted Classroom teaching model in Math teaching” [J]. Information technology Of primary and secondary Education, (2014), No.1,p.21-25 (in Chinese)

[5] Wang Xiaodong, Zhang Chenjing: “Application of Inverted Classroom teaching model in University" (2013), No.8, p.46 (in Chinese)

[6] Hurtubise L, Hall E, Sheridan L, et al. The flipped classroom in medical education: engaging students to build competency[J]. Journal of Medical Education and Curricular Development, (2015), No.2, pp. 35-43.

[7] Jin Ling: "Construction with Chinese characteristics of course teaching" [J]. Information technology education in China, (2013), No.3,p.20 (in Chinese)

[8] Zhang Yueguo, Zhang Yujiang: "Perspective "flip the classroom"” [J]. Information technology Of primary and secondary Education, (2012), No.3,p.9-10 (in Chinese)

[9] Zhong Xiaoliu: "Teaching design research based on inverted classroom in information times" [J]. Open Education Research, (2013), No.2, p.19 (in Chinese)

[10]Liang Leming, Cao Qiaoqiao, Zhang Baohui: "Micro course design mode study" [J]. Open Education Research, (2013), No.1, p.19 (in Chinese)

作者简介: 㚞玉婷 (1981-), 女, 重庆, 教育学硕士, 中教一级, 主要研究方向为外语教育 改革, E-mai1：290021405@qq. com;

罗阳（1979-），男，重庆，副教授、副主任医师、博士生导师（第三军医大学/ 西南大学/中国工程物理研究院), 致力于医学研究生教育教学改革与新型教学模式探索研 究, E-mail: luoyang@tmmu. edu. cn; 入选 “万人计划” 中组部青年拔尖人才、重庆市杰出 青年基金、人社部留学人员择优资助、重庆市五四青年奖章, 承担国家级、省部级及军队课 题 16 项; 获国家科技进步二等奖、“十一五” 军队重大科技成果奖等奖项 11 项。以第一或 通讯作者发表论文 100 余篇, 其中 SCI 论文 17 篇（IF>10 的 2 篇, IF $>5$ 的 9 篇）, 获国际 PCT 专利 3 件（美国、英国、澳大利亚），国家发明专利 14 件，实用新型专利 2 件，软件著 作权 1 件。 